\title{
CONSIDERATIONS ABOUT THE SEVERAL INEQUALITIES IN AN INNER PRODUCT SPACE
}

\author{
NiCUŞOR Minculete
}

Abstract. The aim of this paper is to show new results concerning the Cauchy-Schwarz inequality in an inner product space. We find an improvement of Buzano's inequality and Richard's inequality, which are extensions of the Cauchy-Schwarz inequality.

Mathematics subject classification (2010): Primary 46C05, secondary 26D15, 26D10.

Keywords and phrases: Inner product space, Cauchy-Schwarz inequality, Buzano's inequality, Richard's inequality, Ostrowski's inequality.

\section{REFERENCES}

[1] J. M. AldaZ, Strengthened Cauchy-Schwarz and Hölder inequalities, J. Inequal. Pure Appl. Math., 10 (4), art. 116, 2009.

[2] H. Alzer, A Refinement of the Cauchy-Schwarz Inequality, Journal of Mathematical Analysis and Applications, 168, 1992, 596-604.

[3] M. L. Buzano, Generalizzazione della disiguaglianza di Cauchy-Schwarz, (Italian), Rend. Sem. Mat. Univ. e Politech. Torino 31 (1974) 405-409.

[4] A.-L. CAUChy, Cours d'Analyse de l'École Royale Polytechnique, I ère partie, Analyse Algébrique, Paris, 1821, reprinted by Ed. Jacques Gabay, Paris, 1989.

[5] S. S. DRAGOMIR, A potpourri of Schwarz related inequalities in inner product spaces (II), J. Inequal. Pure Appl. Math. 7 (1) (2006), Article 14.

[6] S. S. DRAGOMIR, Refinements of Buzano's and Kurepa's inequalities in inner product spaces, Facta Universitatis (Niš) Ser. Math. Inform. 20 (2005), 65-73.

[7] I. GavreA, An extention of Buzano's inequality in inner product space, Math. Inequal. Appl., 10 (2007), 281-285.

[8] M. Khosravi, R. DrnovšEK, M. S. Moslehian, A commutator approach to Buzano's inequality, Filomat 26: 4 (2012), 827-832, doi 10.2298/FIL1204827K.

[9] C. LuPU, D. SCHWARZ, Another look at some new Cauchy-Schwarz type inner product inequalities, Applied Mathematics and Computation, 231 (2014), 463-477.

[10] C. P. Niculescu, Inequalities and Identities, A Journey into Mathematics., Mostar, November 11$15,2015$.

[11] A. Ostrows KI, Vorlesungen über Differential-und Integralrechnung, vol. 2, Birkhauser, Basel, 1951.

[12] J. PeČARIĆ, R. RAJIĆ, The Dunkl-Williams equality in pre-Hilbert $\mathbb{C}^{*}$-modules, Lin. Algebra Appl., 425 (2007), 16-25.

[13] D. POPA, I. RAŞA, Inequalities involving the inner product, J. Inequal. Pure Appl. Math. 8 (3) (2007), Article 86, 4 pp.

[14] T. Precupanu, On a generalisation of Cauchy-Buniakowski-Schwarz inequality, Anal. St. Univ. "Al. I. Cuza" Iaşi, 22 (2) (1976), 173-175.

[15] U. RICHARD, Sur des in égalités du type Wirtinger et leurs application aux équationes diffrentielles ordinarires, Colloquium of Anaysis held in Rio de Janeiro, (1972), 233-244. 